\title{
Modeling Prioritized Hard Handoff Management Scheme for Wireless Mobile Networks
}

\author{
BISWAJIT BHOWMIK ${ }^{1}$, POOJA ${ }^{2}$, PIYALI SARKAR ${ }^{2}$, NUPUR THAKUR ${ }^{2}$ \\ ${ }^{1}$ Member of IEEE, IACSIT, IAENG, PASS, IAOE, IAS, and UACEE \\ Department of Computer Science \& Engineering, Indian Institute of Technology Guwahati, 781039, India. \\ b.bhowmik@iitg.ernet.in, biswajitbhowmik@gmail.com \\ ${ }^{2}$ Department of Computer Science \& Engineering, Bengal College of Engineering and Technology, \\ Durgapur, 713212, India \\ pooja.cse2308@gmail.com, piyali.doanju@gmail.com, nupurcse07@gmail.com
}

\begin{abstract}
The channel associated with the current connection serviced by a base station is changed while a call is in progress. Usually, continuous service is achieved by supporting handoff from one cell to another. It is often initiated either by crossing a cell boundary or by deterioration in quality of the signal in the current channel. The existing call is then changed to a new base station. For the traffics which are non stationary at and are away from the servicing base station, the chances of a call to be handed off are increasing. In this paper we propose a scheme $\mathrm{MH}_{2} \mathrm{~S}$ to modeling and implementing a traffic $\bmod e l$ with handoff behavior for wireless mobile networks. The simulation model $\mathrm{MH}_{2} \mathrm{~S}$ with priority is developed to investigate the performance behavior of hard handoff strategy. Novelty of the proposed model $\mathrm{MH}_{2} \mathrm{~S}$ results that it can improve call blocking rate of handoff calls. In addition to this, measurement of blocking probabilities for both originating calls and handoff calls is another impressive achievement of the model.
\end{abstract}

Index Terms - Mobile station, traffic model, arrival rate, departure rate, blocking probability, call blocking rate

\section{INTRODUCTION}

At the present time, traffics (requests and demands for mobile communication facilities) in the upcoming wireless mobile networks (WMNs) are expected to be extremely non stationary [1]. The channel associated with the current connection serviced by a base station $(B S)$ is changed while a call is in progress. Continuous service is achieved by supporting handoff [2] from one cell to the next adjacent cell as the mobile station (MS) moves through the coverage area. The handoff $a \lg$ orithms are able to determine the dynamics of the MSs which move through the WMNs [3][4].

Several competent factors influence to occur a handoff. Two of them has more significant effect on it. One, when a $M S$ moves across a cell boundary from the servicing $B S_{1}$ to another $B S_{2}$. Second, deterioration in quality of the signal in the current channel [2][5][6]. The handoff phenomena in WMNs and mobile cellular communications environment have become progressively more important issue as cell sizes shrink to accommodate an increasingly large $M S S$ in terms of demand for services (traffics) [7]. In this paper, we present a novel lookup on a priority handoff. scheme in a channelized cellular system and WMNs. The term handoff in this paper refers a hard handoff. Another type of handoff is the soft handoff [2]. How a hard handoff is ensured to MSs in $W M N s$ is shown in Figure 1.

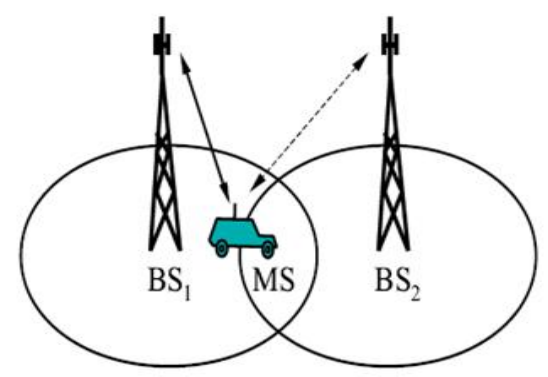

a. Before handoff 


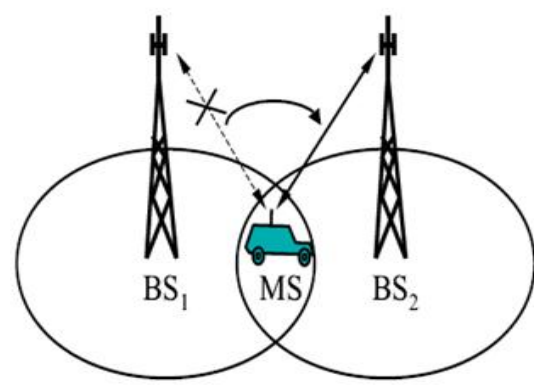

\section{b. After handoff}

Figure 1. Hard handoff between the MS and BSs.

The Figure 1 shows two cases of the current situations of a $M S$ - before and after handoffs. In Figure 1. $a$ we see that a $M S$ is serviced by $B S_{1}$ and it is moving towards $B S_{2}$ without any handoff taken place. In the Figure $1 . b$ we see that a $M S$ has entered in a handoff region. Its services by $B S_{1}$ are cut off and are gained by $B S_{2}$. Eventually a handoff is thus just occurred.

In hard handoff the MS is thus handed off from its current $B S_{1}$ to possible nearest $B S_{2}$. At the moment the $M S S$ leave a cell of a the coverage area of the $B S_{1}$ and enters into a new cell of the coverage area of the $B S_{2}$. In this case, the active set of $M S s$ therefore consists of at most and only one $B S$ at any given time [3].

The decision on the handoff to be taken place from one cell to the other is based on various criteria that take into account of channel degradation considerations too [5]. However, the initial (and most important) trigger for a handoff is generally based on pilot signal strengthmeasurements taken for a $M S$ at the underlying $B S$. This $B S$ is also known as mobile ter min al (MT) [8][9]. The $S_{2} B P Q$ model [8] has taken into account the performance of handoff behavior on the basis of received or relative signal strength (RSS) [5][6][10][11]. The simulated results suggest that a handoff point (the maximum allowable radial distance from $B S_{1}$ at which $M S s$ possibly gets serviced by another nearest $B S_{2}$ instead of its current servicing $B S_{1}$ ) for a $M S$ depends on various parameters that have direct impact on this $R S S$ as determined by Equation (1) in [8][10]. Suppose the radial distance of $M S s$ from a $B S(M T)$ is $r$. Calls are generally two types - originating calls, andhandoff calls. In this paper we suggest a simulation model $\mathrm{MH}_{2} \mathrm{~S}$ with priority handoff scheme for modeling and implementing a traffic mod $e l$, and evaluating blocking probabilities of originating calls $\left(B_{O}\right)$, and handoff calls $\left(B_{H}\right)$ with the selected traffic $\bmod e l$ [2][8]. All these are performed once we have completed the computation of arrival rate of $h a n d o$ calls $\left(\lambda_{H}\right)$ of the traffic mod $e l$ selected in this paper.

The cellular structure which is considered in all the cases such as $S_{2} B P Q$ [8], EATM [13], and $\mathrm{MH}_{2} \mathrm{~S}$ models is shown in Figure 2. This is a well known and well efficient cellular classification of the coverage of a $M T$ and is used in practice. The Figure 2 shows a part of the total cellular configuration (coverage area of a servicing $B S$ ). It is actually a segment taken at $120^{\circ}$ orientation of the area.

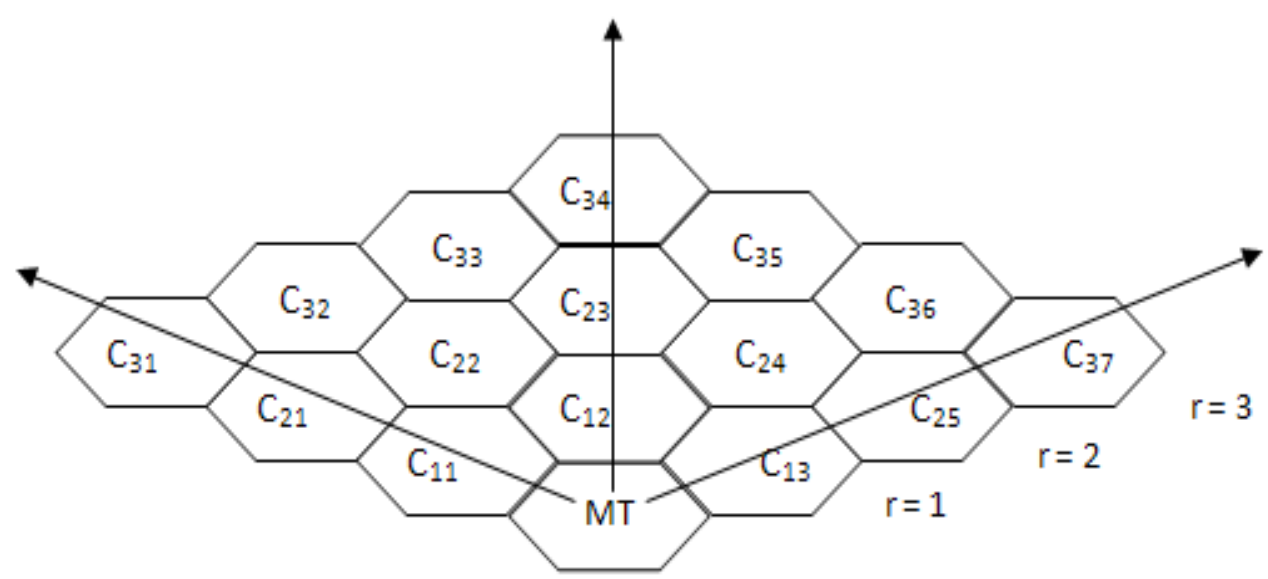

Figure 2. A typical cellular configuration of underlying MT for $r=3$. 
We organized our paper as follows. First, we start with some preliminary assumptions which have been assumed in Section II. Second, the proposed work has been elucidated in section III. In this section we have selected a traffic mod el followed by modeling it. The model is best suitable for the assumptions would be taken in preceding Section.. We are here able to derive some suitable mathematical expressions for several attributes of the selected traffic mod $e l$. Third, performance of the proposed model $\mathrm{MH}_{2} \mathrm{~S}$ is appraised with simulation in Section IV. The simulation has been shown both in numerical and corresponding graphical. The paper is over and completed drawing some remarkable conclusions. This has been given in Section V.

\section{PRELIMINARIES}

We have already known that the $R S S$ measurement is one of the most common criteria to initiate a handoff [5][6]. We assumed two base stations mod el in [3][8][11][12] as primary objective of a handoff algorithm that provides a good signal quality. We consider only that portion of the trajectory on which the signals received from the two $B S S$ are the strongest. Generally, a high $R S S$ means good signal quality, so the handoff to another $B S$ cannot be occurred unnecessary, because the $M S$ is being served well by the current servicing $B S$ and on a handoff taken place, all the services must be quit from the current $B S$. We restrict our analysis to short radial distance $r$ horizons over which a $M S$ moves from one radial distance to another with fixed velocity in any direction (away from current $B S$, towards $B S$, or along same radial level from current $B S$ ) with equal probability of movement. Movements of $M S s$ in cells are shown in Figure 3 [14]. However, movements of the $M S s$ are unrestricted and random in nature.

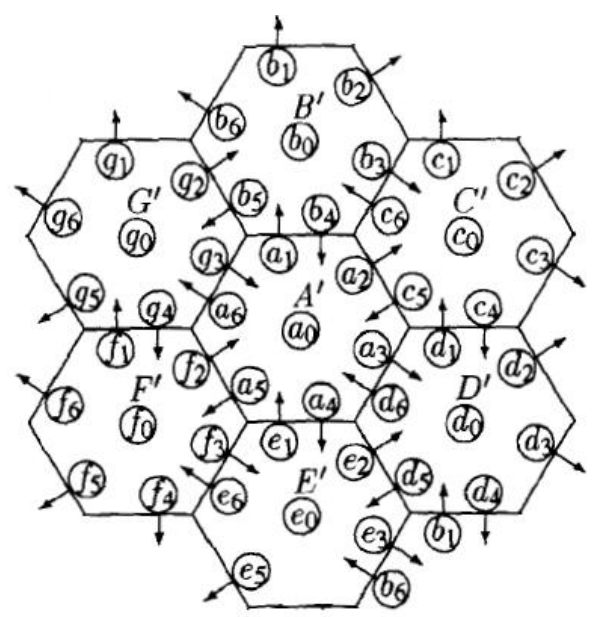

Figure 3. Movements of the MSs.
Although the MSs move randomly in the coverage area. But a handoff is only possible as already we know when a $M S$ either crosses a cell boundary merely or its $R S S$ is lower than threshold value. Crossing a cell boundary may be possible three ways. First, when MSs move from a cell in radial distance $r$ to a cell at radial distance $(r+1)$. Second, this case is exactly opposite to the previous one. That means $M S s$ in this case move from a cell in radial distance $r$ to a cell at radial distance $(r-1)$. Third, $M S s$ in this case move from a cell to another along the same radial distance $r$. However, third case is very less responsible for a handoff to be happened since $R S S$ of the underlying $M T s$ for $M S s$ remains same. Second case is also less responsible for a handoff to be happened since $R S S$ of the underlying $M T s$ for $M S s$ become strengthen since the $M S s$ in this case move towards their servicing $M T$. The three cases of movements of the MSs have shown graphically. The Figure 4 represents them. Here any node numbered as $i j, i, j \in N$ in Figure 4 represents any cell $C_{r j}$ of the cellular structure shown in Figure 2.

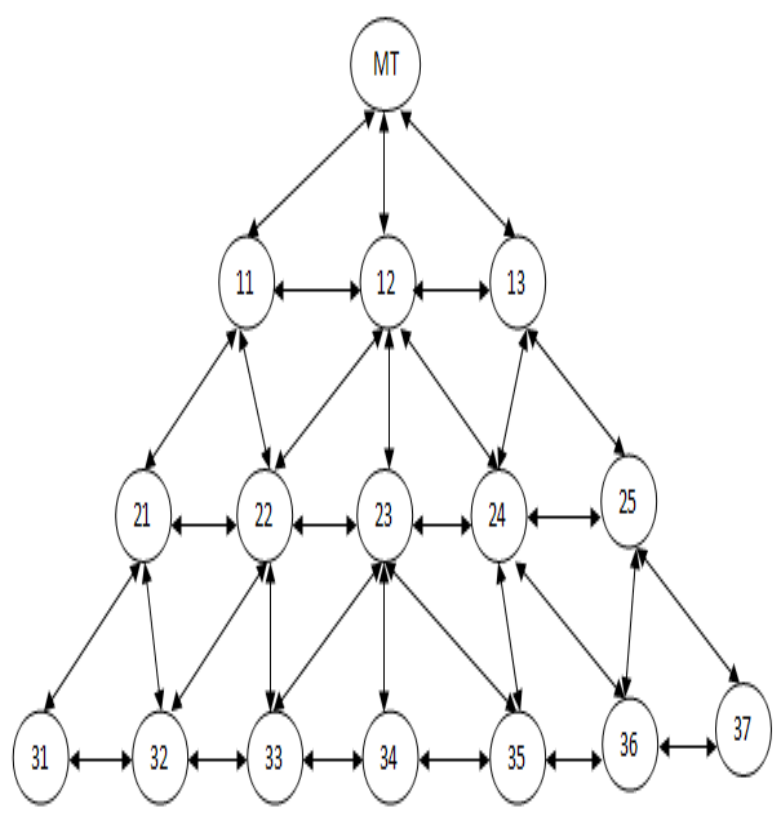

Figure 4. Graphical presentation of movements of MSs.

The MSs also called mobile callers (MCs) are evenly distributed over the coverage area of a $B S$ as shown in Figure 3. That means a basic system model assumes that the new call origination rate is uniformly distributed over the mobile service area $[2][3][4][5][8][9][10][11][15][16][17]$. But it is seen that $M S s$ arrive in a $B S$ randomly. This means requests of $M S s$ are made non-uniformly. We assume these requests are made according to Poison distributions [18]. 


\section{PROPOSED WorK}

In next-generation wireless systems and $W M N s$ it is important for the $M S S$ to ensure that the system is guaranteeing their needed requirements. These basic requirements would improve the quality of service $(Q o S)$ provided by WMNs . Therefore, a proper traffic management scheme is required to effectively manage the ever increasing traffics $(M S s)$ which are non stationary at and is on the way away from the servicing $B S$ in the system. There are many proposals to solve the dilemma [1][19]. Our approach provides high precise location and tracking of $M S s$ by exploiting advanced traffic mod els. Some of these we have studied in [7][8][15][20]. Here we have extended our previous work [8][13] with El-Dolil et al.'s traffic mod el [2]. Our proposed model $\mathrm{MH}_{2} \mathrm{~S}$ takes more advantages over previous model described in [7][8][13][15].

The major functionalities of the proposed model $\mathrm{MH}_{2} \mathrm{~S}$ have been classified into following sub areas. First, we determine arrival rate $\left(\lambda_{O}\right)$ of originating calls. Second, we determine depurture rate $(\mu)$ of MSs that gets serviced by their servicing $B S$. Third, we select a suitable traffic mod $e l$ for implementation. Four, we have chosen a scheme for handoff based on priority. Five, we allocate some channels to originating calls, handoff calls, and handoff requests. Last, more influencing factor of the model call blocking rate $(C B R)$ is determined.

\section{A. Determination of $\lambda_{O}$}

Number of MSs (traffic density [18]) varies location to location. And this location on the contrary affects arrival rate $\lambda o$ of originating calls from $M S s$ to their underlying $B S$. Likewise number of $B S s$ are also varied. Assuming distance D [4][17] between nearby two $B S s$ is $1-3 \mathrm{~km}, \lambda o$ has been determined here similar to $S_{2} B P Q$ model [2] [8] as:

$$
\lambda o \approx \frac{\text { Total Subscribers }(S) \text { in the Re gion }}{\text { Total Number of MTs }(X)}
$$

\section{B. Determination of $\mu$}

The model $S_{2} B P Q$ [8] might be competent of providing services to all (may be infinite number) $M S s$ with no or least waiting time after initiating a call (request for service). Thus, departure rate $\mu$ (number of $M S s$ get serviced in unit time) should be at least equal to $\lambda o$ such that waiting time for getting services generally becomes zero or very less. Exploiting Poison distributions [18], and the traffic intensity factor $\rho$ defined as $\lambda / \mu$ lies in the range $[0-1]$, departure rate $\mu$ has been considered similar to $S_{2} B P Q$ and EATM models [8][13] as:

$$
\begin{aligned}
& 0 \leq \rho \leq 1 \\
& \Rightarrow \quad 0 \leq \lambda_{O} / \mu \leq 1 \\
& \Rightarrow \quad 0 \leq \lambda_{O} \leq \mu
\end{aligned}
$$

However, $\mu$ should be much greater than $\lambda_{O}$ so that the MSs get services on their request immediately. Waiting calls are enqueed in a busy list. A call in this list does exist for a little time quanta. When a time quanta is over and the call is not scheduled for services, it is then dropped from the list.

\section{Selection of Traffic Model}

Every cell in cellular network architecture is served by a $B S$. The $B S S$ are connected together by using a wireless network. Establishment of a traffic mod $e l$, in cellular system, is more imperative before analyzing the performance of the system [7][8]. Several traffic mod els [2] have been established on basis of making different assumptions about user mobility. We measure the performance of handoff $a \lg$ orithms in terms of the expected rate of handoff $\left(\lambda_{H}\right)$. This is one of the parameters used to analyze handoff performance [5][9]. We consider El-Dolil et al.'s traffic mod $e l$ [2] which is shown in Equation (5). The selected traffic mod $e l$ is represented in terms of performance parameter i.e. the arrival rate of handoff calls $\left(\lambda_{H}\right)$. The $\lambda_{H}$ is then given by:

$\lambda_{H}=\left(R_{c j}+R_{s h}\right) P_{h i}+R_{s h} P_{h h}$

Where,

$R_{c j}=$ average rate of total calls carried in cell $j$.

$R_{s h}=$ the rate of successful handoffs.

$P_{h i}=$ the probability that a mobile station needs a handoff in cell $i$. 
$P_{h h}=$ probability that a call that has already been handed off successfully would require another handoff.

The model has been chosen as underlying implementation model based on some basic assumptions. One, the highway is segmented into cellular structures (microcells) with small $B S s$. Second, along the highway mobile radio signals that are radiating are cigar-shaped [2][8]. We have derived general mathematical expressions for these parameters of the Equation (5) as follows.

1) Determination of $R_{c j}$ : Consider Figure 2 again. At radial distance $r=1$ from servicing $B S(M T)$, number of cells is 3 . In the same fashion at $r=2,3 \ldots R_{\max }$ number of cells are $5,7 \ldots\left(2 \times R_{\max }+1\right)$ respectively. Thus, $R_{\max }$ represents maximum radial distance or handoff po int [8]. In general, total cells $\mathrm{N}$ under the coverage area of a typical $B S$ is given by:

$$
N=\sum_{r=1}^{R_{\max }}(2 r+1)
$$

However traffic density is not uniform. For better services, a $B S$ should have some number of $M S s$ to make it busy and the $B S$ should not be overloaded some time. Although this not the actual cases. Because a $B S$ may be overloaded some time or may goes down with performances. Average number of $M S s, S u b s$ in any cell $j$ at any $r$ from its $B S$ (represented as $C_{r j}$ ) is obtained as:

$$
\text { Subs }=\frac{\text { Total Number of MSs }(S)}{\text { Total Number of Cells }(N)}
$$

The Subs are not actually $M S s$ rather they are meant by the $M S s$ who have already made requests. The $M S s$ are allowed making any number of requests.

Let us assume a term that an average number of calls (requests) originated by subscriber (MS) is calls per day per subscriber (CPD). Thus
$R_{c j}$ can be determined at any particular radial distance $j$ from the current $B S$ as:

$$
R_{c j}=\prod(S u b s, C P D,(2 j+1)), j=1,2 \ldots R_{\max }
$$

2) Determination of $R_{s h}$ : The MSs are non stationary. When a $M S$ is moving away from its $B S$, its $R S S$ value is decreasing. Therefore, a $M S$ has a chance to be handed off and probably is handed off from serving $B S_{1}$ to another $B S_{2}$ when this $R S S$ value gets decreased below at least $50 \%$ of its original strength value [8]. The $R S S$ is sampled at discrete time instants $t_{j}=k t_{s}$, where $t_{s}$ is sampling time and corresponding sampling interval in distance is $d_{s}=v t_{s}$. . Here, $v$ is constant velocity of a $M S$ [12]. Thus, at the radial distance $R_{\max }$ from the current $B S$, a handoff for the $M S$ occurs first time. However, before a call $(M S)$ is handed over from $B S_{1}$ to another $B S_{2}$ it has to travel a radial level $R_{\text {max }}$. Therefore, Subs are gradually increased when $M S s$ move to $r$ from $(r+1)$.

We assume for simplicity that two-third movement of the $M S s$ take place to immediate upper radial level from current radial level. Few of them may come back to their starting radial distance. Therefore total number of $M S s$ moved away from their $B S$ are effectively less, We assume that one-third of the $M S S$ move back to its immediate lower radial level from current radial level. These movements are applicable to all the radial level. Thus, taking effect of both in - just upper and lower radial levels, $R_{s h}$ at any level $r$ from its nearest $B S$ has been determined in Equation (9) as:

$$
R_{s h}=\left\{\begin{array}{l}
\prod\left(\left(\frac{2}{3}(2 r+1)+\frac{1}{3}(2 j+1)\right), \text { Subs }\right), r=1, \quad j=r+1 \\
\prod\left(\left(\frac{1}{3}(2 i+1)+\frac{2}{3}(2 r+1)+\frac{1}{3}(2 k+1)\right), \text { Subs }\right), r=2,3 \ldots . . R_{\max }, i=r-1, \quad k=r
\end{array}\right.
$$


3) Determination of $P_{h i}$ : Movements of the $M S S$ are either towards or away from current serving $B S$ and are primarily responsible for handoff mechanism to be taken place. Every $M S$ has same opportunity to be handed over. Let us choose any mobile station $M S_{r}$ at any radial distance $r$, $1 \leq r \leq R_{\max }$ from its underlying $B S$. Therefore, the probability of selection of $M S_{r}$ could be represented as:

$$
P\left(M S_{r}\right)=\frac{1}{2 r+1+\delta}
$$

Where $\delta=$ a constant factor assumed as the effect of adjacent cells (left and right most) at any $r$.

We consider the value of $\delta$ equals to 2 when a $M S$ moves towards $B S_{1}$ and 0 when it moves towards $B S_{2}$. The $P\left(M S_{r}\right)$ can be computed in two ways. One, in - ward handoff probability $P_{h_{i}}(\downarrow)$ in a cell $i$. Second, out - ward handoff probability $P_{h_{i}}(\uparrow)$ in a cell $i$. These names are given according to the movements of $M S s$. The $P_{h_{i}}(\downarrow)$ is likely to be happened when $M S s$ move towards their servicing $B S$. And, the $P_{h_{i}}(\uparrow)$ is likely to be happened when the $M S s$ move away from their servicing $B S$. Here, both $P_{h_{i}}(\downarrow), \quad$ and $\quad P_{h_{i}}(\uparrow)$ are int er-level handoff probabilities. However, an actual handoff for a $M S$ takes place at $r=R_{\max }$. Another interesting thing is that the probability $P_{h_{i}}(\downarrow)$ decreases the chances of handoff to be occurred till $r \geq 1$ while the probability $P_{h_{i}}(\uparrow)$ enhances the chances of handoff to be occurred till $r \leq R_{\max }$. Assuming initial values of $P_{h_{i}}(\downarrow)$, and $P_{h_{i}}(\uparrow)$ are zero, We compute them as:

$$
P_{h_{i}}(\downarrow)=\frac{1}{3} \sum_{r}\left(P_{h_{i+1}}(\downarrow)+\frac{1}{2 i+3}\right), i=R_{\max } \ldots 2,1
$$

And,

$$
P_{h_{i}}(\uparrow)=\frac{1}{3} \sum_{r}\left(P_{h_{i-1}}(\uparrow)+\frac{1}{2 i+1}\right), i=1,2 \ldots R_{\max }
$$

Now, a handoff point the maximum radial distance of a $M S$ from its servicing $B S$ ) is at $r \leq R_{\max }$. Before reaching a $M S$ to a handoff point it may have either $P_{h_{i}}(\downarrow)$ or $P_{h_{i}}(\uparrow)$. Therefore, at any level $r, 1 \leq r \leq R_{\max }$, the probability $P_{h i}$ that a $M S$ needs a handoff in cell $i$ can thus be urged as below.

$$
P_{h i}=1-\left(P_{h_{i}}(\uparrow)+P_{h_{i}}(\downarrow)\right), r=1,2 \ldots . R_{\max }
$$

4) Determination of $P_{h h}:$ We have exploited the property that two-third of the $M S s$ at radial level $r$ move to immediate upper level $(r+1)$ and one-third of the $M S s$ at radial level $r$ move to immediate lower level $(r-1)$. Some of the MSs under $B S_{1}$ at the handof point may move to $\left(R_{\max }+1\right)$ which is similar to $R_{\max }$ from $B S_{2}$. Second handoff may occur when $M S S$ eventually come back to $R_{\max }$ with respect to $B S_{1}$. Therefore, the probability of next handoff $P_{h h}$ of a $M S$ may be determined as -

$$
P_{h h}=\frac{1}{3}\left(\frac{2}{3} \sum_{r=1}^{R_{\max }} \frac{1}{3^{r}}+\frac{1}{3^{R_{\max }}}\right)
$$

\section{Priority Handoff Scheme}

A handoff request is generated in a cell when a $M S$ approaches the cell from a neighboring cell with significant signal strength. Priority is set to handoff requests and their types. Some channels are necessarily assigned in a cell. These assigned channels may be exclusive or shared. Suppose, $S_{R}$ channels have been assigned exclusively for handoff calls out of $S$ channels . And, both originating calls and handoff requests share the remaining $S_{c}=S-S_{R}$ channels. An originating call call is blocked if the number of available channels in the cell is less than or equal to $S_{R}$. Similarly a handoff request is blocked if no channel is available in the target cell. The system model for the channels sharing is shown in Figure 5 [2][7]. 


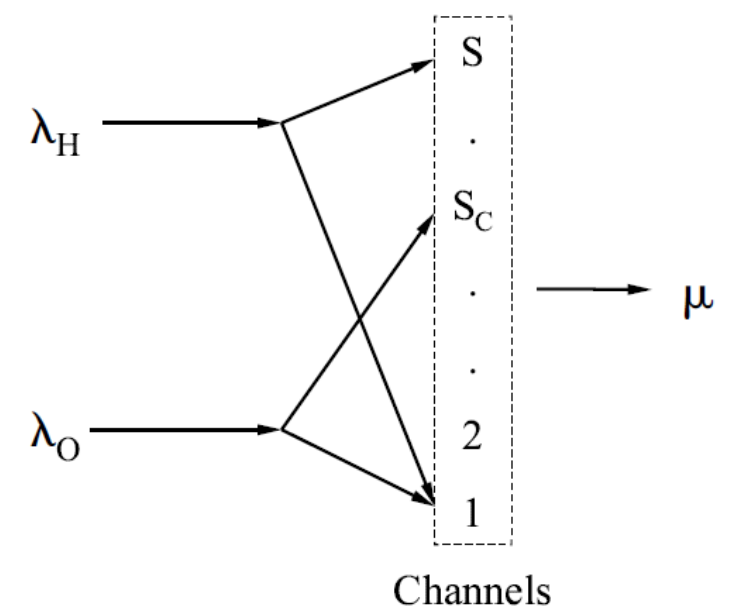

Figure 5. System model with priority for handoff call.

Two important parameters can be derived from this allocation of channels. These are blocking probabilities $B_{O}$ of originating calls, and $B_{H}$ of handoff requests [2][7][8] respectively. They have been determined by Equations (15), and (16) with the steady-state probability $P(i)$ [2] respectively.

$B_{O}=\sum_{i=S_{C}}^{S} P(i)$

And,

$B_{H}=\frac{\left(\lambda_{O}+\lambda_{H}\right)^{S_{C}} \lambda_{H}^{S-S_{C}}}{S ! \mu^{S}} P(0)$

Here $P(0)$ [2][7] states steady state probability when the system is in state " 0 ". We define the state $i, i=0,1,2 \ldots . . S$ of a cell as the number of calls in progress for the $B S$ of that cell.

\section{E. Channel Allocation Scheme}

A new call holds the channel until the call is completed in the cell or it move out of the cell. A successful handoff call holds the channel until the call is completed in that cell. Thus, handoff call is admitted until all channels are busy [21]. In the evaluation of handover (handoff) performance, number of channels to be allocated for handoff requests, and originating calls are exclusively important very much along with other factors such as $R S S, R_{\max }$ etc [2][22]. Already we have seen that how some channels $S$ are necessarily assigned in a cell forhandoff calls, handoff requests, and originating calls. Although all the channel allocation schemes are facing the same challenge that how the channels could be distributed (allocated) effectively to these calls. Because the amount of channels in terms of frequency ranges is fixed.

\section{F. Determination of Call Blocking Rate}

A $M S$ when initiates a call, it generally expects to be get serviced immediately. Types of requests are different. Before a request gets serviced, it must be enqueed in priority queue [8][23]. Afterward a call gets serviced by its current $M T\left(B S_{1}\right)$ taking advantage of Splay operations on the Splay Tree [24] implementation of the priority queue generated in [8]. The selection of a call in the Splay Tree, follows SIRO queuing principle [18]. So, more and more number of cells are getting services in a cell. At particular radial level $r$, $r=1,2 \ldots R_{\max }$ and a specific time instant, number of calls blocked (enqueed) for availing services i.e. call blocking rate $\left(C B R_{r}\right)$ [25] could be decided as:

$$
C B R_{r}=\frac{1}{3} \prod\left(R_{c j_{r}}, B_{O},(2 r+1)\right)
$$

We will show that this $C B R_{r}, r=1,2 \ldots R_{\max }$ will be increasing with $r$ increases.

\section{SIMULATION WORK}

The parameters used for simulation are commonly used to analyze handoff performances. We simulated our model $\mathrm{MH}_{2} \mathrm{~S}$ in MATLAB Version 7.6.0.324 (R2008A). Numerical values of the fundamental parameters for handover initiations, $\lambda_{o}$, and $\mu$ are based on COAI REPORT [26][27] developed for beloved Megacity Kolkata. These parameters are set as $\lambda_{o}=1991$, and $\mu=2212$ [8]. Exploiting these numerical on all the Equations (5) through (17) whenever necessary are assessed. Here we have shown two observations. We assume that the shadow fading effect $\zeta(\mathrm{r})$ is $\log (\mathrm{r})$ in all the observations.

Observation I: Let us suppose that a $M C$ makes at least 5 requests per day $(C P D)$. We assumed $\mathrm{C}=0$, and $\eta=20$ in Equation (1) [8] and we found $R_{\max }=14$. Using Equation (6) and Equation (7), total number of cells $\mathrm{N}$ and average number of $M S S$ in any cell Subs are found 224 and 2.9632 respectively. Other values are shown in Table I. 
Table I: Simulation of $\mathrm{MH}_{2} \mathrm{~S}$ for CPD $=5$.

\begin{tabular}{|c|c|c|c|}
\hline Parameters & \multicolumn{3}{|c|}{$\begin{array}{c}\text { Numerical Outcomes Under Current } \\
\text { Base Station Level Wise }\end{array}$} \\
\hline \multirow{5}{*}{$R_{c j}$} & 44.44742 & 74.07904 & 103.7107 \\
\hline & 133.3423 & 162.9739 & 192.6055 \\
\hline & 222.2371 & 251.8687 & 281.5004 \\
\hline & 311.132 & 340.7636 & 370.3952 \\
\hline & 400.0268 & 429.6584 & \\
\hline \multirow{5}{*}{$R_{s h}$} & 10.86493 & 19.75441 & 27.65617 \\
\hline & 35.55794 & 43.4597 & 51.36147 \\
\hline & 59.26323 & 67.165 & 75.06676 \\
\hline & 82.96852 & 90.87029 & 98.77205 \\
\hline & 106.6738 & 114.5756 & \\
\hline \multirow{5}{*}{$P_{h i}$} & 0.80067 & 0.83163 & 0.86666 \\
\hline & 0.89321 & 0.91206 & 0.92555 \\
\hline & 0.93549 & 0.94306 & 0.94903 \\
\hline & 0.95388 & 0.95797 & 0.96166 \\
\hline & 0.96559 & 0.97131 & \\
\hline$P_{h h}$ & & 0.11111 & \\
\hline \multirow{5}{*}{$\lambda_{H}$} & 28.09555 & 47.37267 & 68.98609 \\
\hline & 91.29296 & 113.8331 & 136.4347 \\
\hline & 159.0444 & 181.6503 & 204.2531 \\
\hline & 226.8593 & 249.4859 & 272.1833 \\
\hline & 295.1101 & 318.7731 & \\
\hline$\lambda_{O}$ & & 22.8851 & \\
\hline$\mu$ & & 25.4278 & \\
\hline$B_{O}$ & & 0.75684 & \\
\hline \multirow{5}{*}{$B_{H}$} & 0.089776 & 0.12517 & 0.17025 \\
\hline & 0.21778 & 0.26604 & 0.31441 \\
\hline & 0.36272 & 0.41096 & 0.45914 \\
\hline & 0.50731 & 0.55555 & 0.60407 \\
\hline & 0.65355 & 0.70618 & \\
\hline \multirow{5}{*}{$C B R_{r}$} & 33.639646 & 93.44346 & 183.14918 \\
\hline & 302.75681 & 452.26635 & 631.67779 \\
\hline & 840.99114 & 1080.2064 & 1349.3236 \\
\hline & 1648.3426 & 1977.2636 & 2336.0865 \\
\hline & 2724.8113 & 3143.438 & \\
\hline
\end{tabular}

The graphical representations of the parameters $\lambda_{H}$, $B_{O}, B_{H}$, and $C B R_{r}$ produced in Table I for Observation I , have been shown in the Figures 6 though 9 respectively.

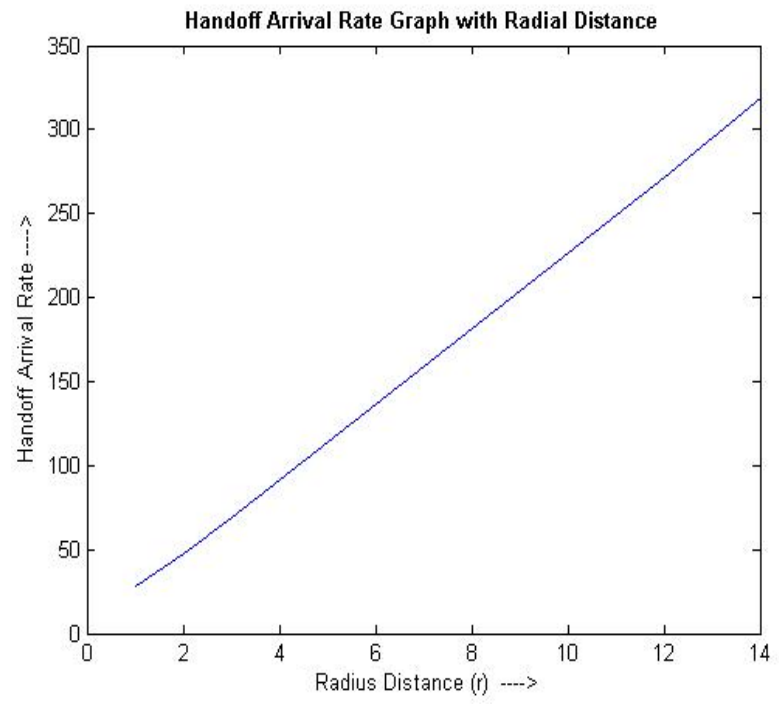

Figure 6. Arrival rate of handoff requests.

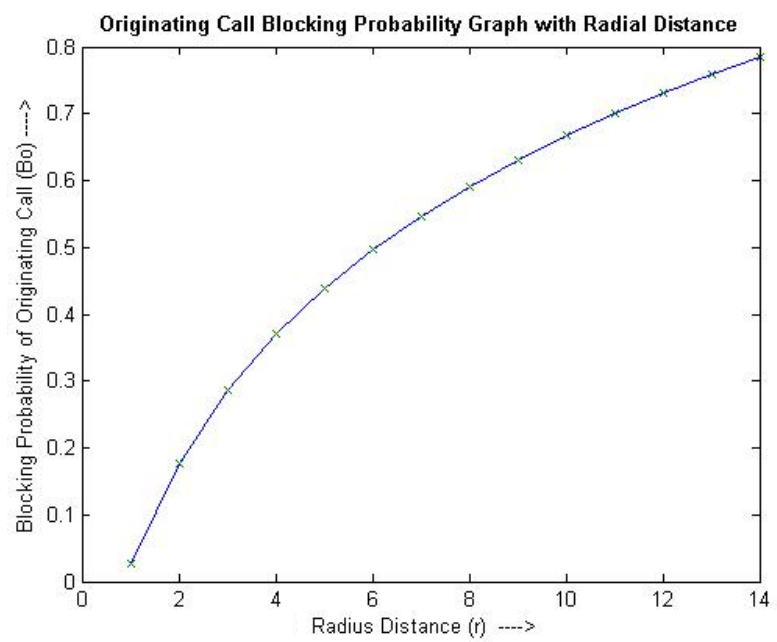

Figure 7. Growth of $\mathrm{B}_{\mathrm{O}}$ for originating calls.

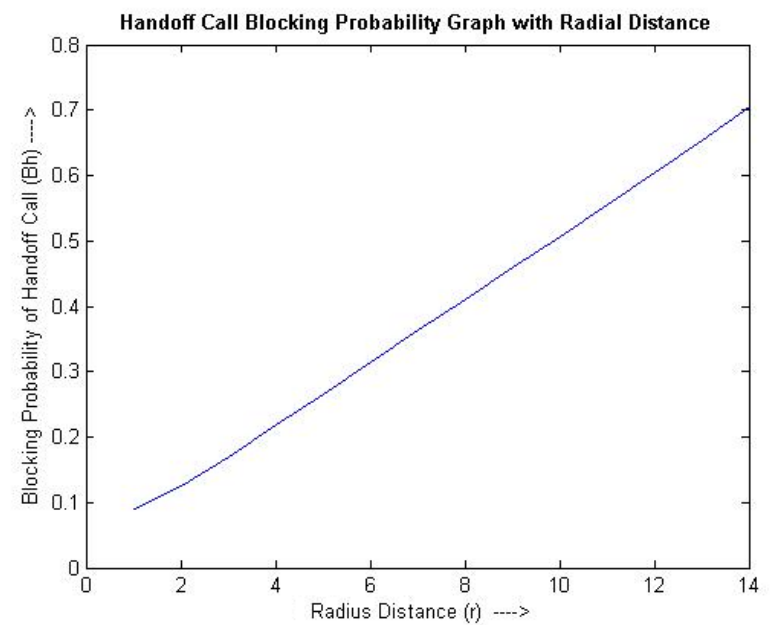

Figure 8. Growth of $\mathrm{B}_{\mathrm{H}}$ for handoff calls. 


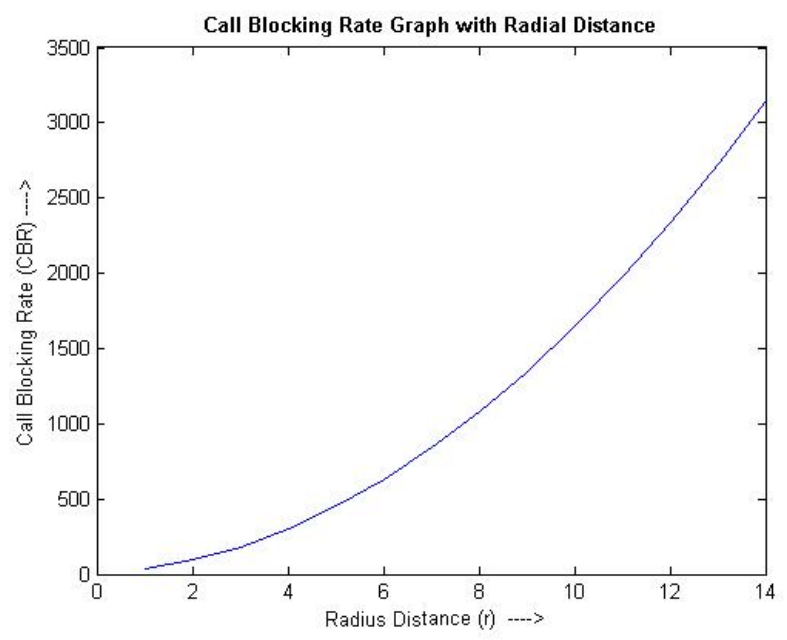

Figure 9. Growth of call blocking rate.

Observation II: Let us suppose that a $M C$ makes at least 6 requests per day $(C P D)$. In the same way (simulation made in Observation I) assuming $\mathrm{C}=0$, and $\eta=21$ in Equation (1) [8], we get $R_{\max }=12$. Similarly $\mathrm{N}$ and $S u b s$ are obtained as 168 and 3.9509 respectively from Equation (6) and Equation (7) respectively. Other values are shown in Table II.

Similarly, the graphical representations of the parameters $\lambda_{H}, B_{O}, B_{H}$, and $C B R_{r}$ produced in Table II for this Observation II , have been shown in the Figures 10 though 13 respectively. First we showed Figure 10 which represents the parameter $\lambda_{H}$. Other Figures 11 through 13 have been shown after Table II.

The $C P D$ values are increased in both the observations by 1 . Other higher values could be assigned to $C P D$. However, in general it is seen that $C P D$ values are not changed suddenly. These values are based on the statistics collected from certain percentage of general people.

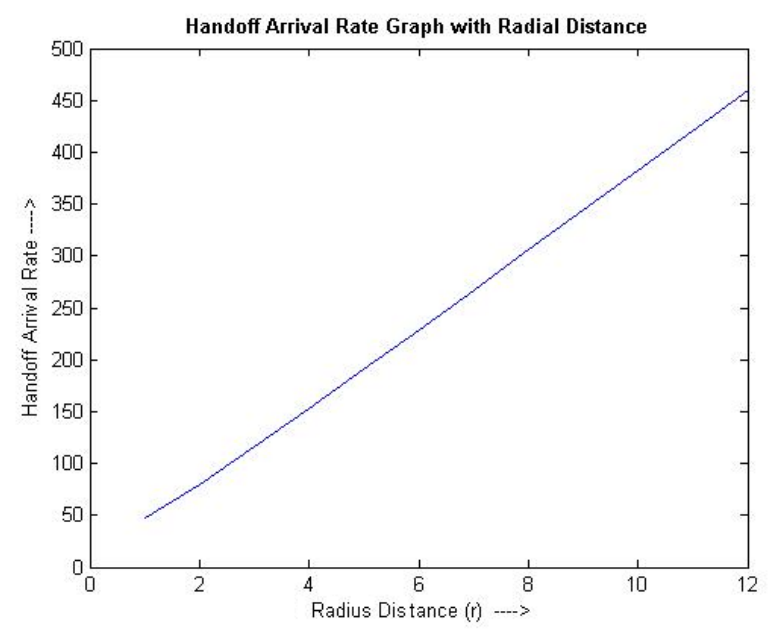

Figure 10. Arrival Rate of handoff requests.
Table II: Simulation of $\mathrm{MH}_{2} S$ for CPD $=6$.

\begin{tabular}{|c|c|c|c|}
\hline Parameters & \multicolumn{3}{|c|}{$\begin{array}{c}\text { Numerical Outcomes Under Current } \\
\text { Base Station }\end{array}$} \\
\hline \multirow{4}{*}{$R_{c j}$} & 71.11588 & 118.5265 & 165.937 \\
\hline & 213.3476 & 260.7582 & 308.1688 \\
\hline & 355.5794 & 402.99 & 450.4006 \\
\hline & 497.8111 & 545.2217 & 592.6323 \\
\hline \multirow{4}{*}{$R_{s h}$} & 14.48657 & 26.33921 & 36.8749 \\
\hline & 47.41059 & 57.94627 & 68.48196 \\
\hline & 79.01764 & 89.55333 & 100.089 \\
\hline & 110.6247 & 121.1604 & 131.6961 \\
\hline \multirow{4}{*}{$P_{h i}$} & 0.80067 & 0.83163 & 0.86666 \\
\hline & 0.89321 & 0.91206 & 0.92555 \\
\hline & 0.93551 & 0.94313 & 0.94922 \\
\hline & 0.95444 & 0.95964 & 0.96668 \\
\hline$P_{h h}$ & & 0.11111 & \\
\hline \multirow{4}{*}{$\lambda_{H}$} & 46.95075 & 79.59185 & 115.9499 \\
\hline & 153.4849 & 191.4158 & 229.4519 \\
\hline & 267.5049 & 305.5608 & 343.6436 \\
\hline & 381.8374 & 420.4091 & 460.2128 \\
\hline$\lambda_{O}$ & & 26.5467 & \\
\hline$\mu$ & & 29.4963 & \\
\hline \multirow{4}{*}{$B_{O}$} & 0.032694 & 0.19616 & 0.31293 \\
\hline & 0.40374 & 0.47805 & 0.54092 \\
\hline & 0.59541 & 0.64349 & 0.68651 \\
\hline & 0.72543 & 0.76097 & 0.79366 \\
\hline \multirow{4}{*}{$B_{H}$} & 0.12074 & 0.17735 & 0.24776 \\
\hline & 0.32228 & 0.39813 & 0.47429 \\
\hline & 0.55046 & 0.6266 & 0.70281 \\
\hline & 0.77945 & 0.85757 & 0.94058 \\
\hline \multirow{4}{*}{$C B R_{r}$} & 58.126376 & 161.46216 & 316.46583 \\
\hline & 523.13739 & 781.47684 & 1091.4842 \\
\hline & 1453.1594 & 1866.5025 & 2331.5135 \\
\hline & 2848.1924 & 3416.5392 & 4036.5539 \\
\hline
\end{tabular}




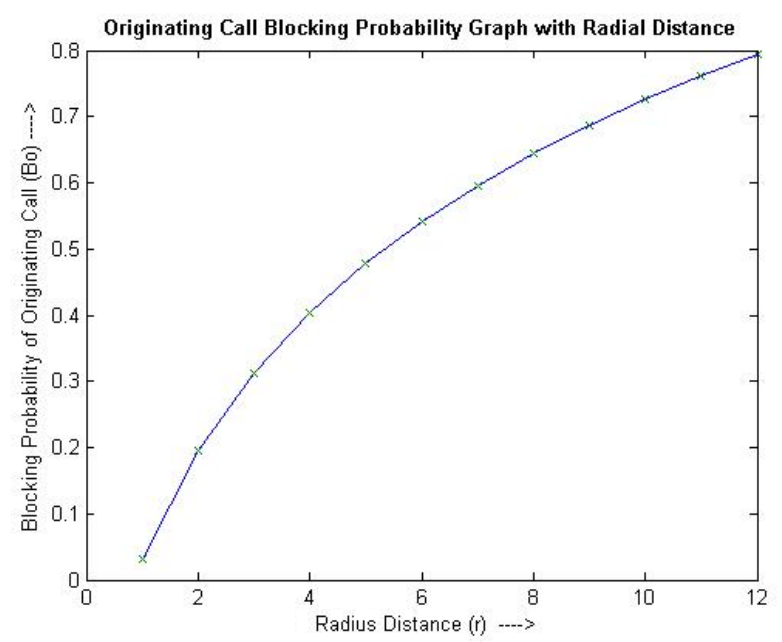

Figure 11. Growth of $\mathrm{B}_{\mathrm{O}}$ for originating calls.

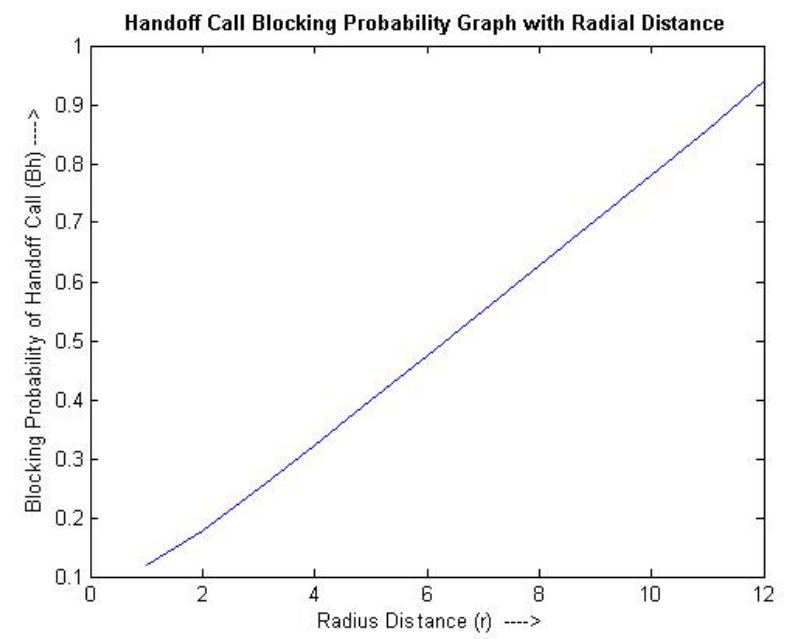

Figure 12. Growth of $\mathrm{B}_{\mathrm{H}}$ for handoff calls.

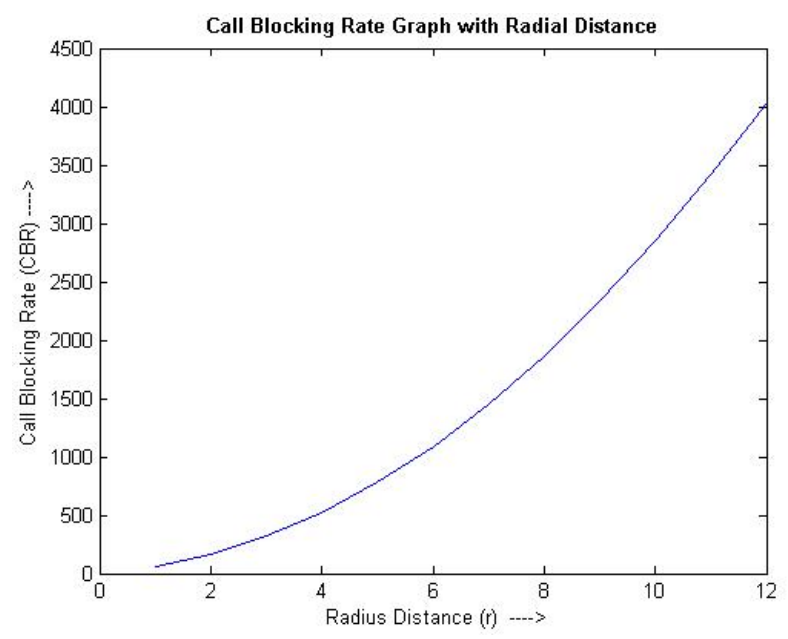

Figure 13. Growth of call blocking rate.

\section{CONCLUSIONS}

To the best of our knowledge, it is the first time that an explicit mathematical derivations have been proposed to calculate arrival rate of handoff calls $\lambda_{H}$ when the MSs are mobile. We are able to present an easy method that evaluates priority scheme on selection of a suitable traffic mod el analytically. Simulation results show that our algorithm performs better than some existing handoff $a \lg$ orithms. The proposed model $\mathrm{MH}_{2} \mathrm{~S}$ can achieve satisfactory number of handoffs taken place on an average. Compared with other handoff $a \mathrm{lg}$ orithms, the only overhead of the proposed algorithm is proper allocation of channels. Therefore, our algorithm can improve handoff performance effectively at the cost of very marginal overhead comparatively with less number of channels. It is observed that increasing average number of calls per $M S s$ per day i.e. CPD helps to improve the $B_{O}$ for both handoff requests, and originating calls. And these values are nearer to their actual values. Another achievement of the proposed model is working out of rate of blocked calls $(C B R)$ in addition.

\section{ACKNOWLEDGEMENTS}

First author would like to convey his heartily gratitude to Mr. Parag Kumar Guhathakurta, Mr. Sanjib Sadhu , Assistant Professors, Department of Computer Science \& Engineering, National Institute of Technology Durgapur, and Dr. S. Nandi, Professor, Dr. G. Barua, Professor, and Dr. S. Ranbir Singh, Assistant Professor, Department of Computer Science \& Engineering, Indian Institute of Technology Guwahati for their valuable suggestions, remarks, criticisms, and comments towards quality improvement of this paper. Other authors would also like to express their thankfulness to their friends especially classmates of Department of Computer Science \& Engineering, Bengal College of Engineering and Technology, Durgapur for their dear inspirations and moral supports.

\section{REFERENCES}

[1] N. Mohan, T. Ravichandran, “An Efficient Multiclass Call Admission Control and Adaptive Scheduling for WCDMA Wireless Network", European Journal of Scientific Research, Vol. 33 No. 4, 2009, Pp.718-727.

[2] Ivan Stojmenovic, Qing-an Zeng and Dharma P. Agrawal, Handbook of Wireless Networks and Mobile Computing, John Wiley \& Sons, Chapter 1, 2002.

[3] Alexe E. Leu, Brian L. Mark, "A Discrete-Time Approach to Analyze Hard Handoff Performance in Cellular Networks", IEEE Transactions on Wireless Communications, Vol. 3, No. 5, 2004, Pp: 1721 1733. 
[4] S. A. Mawjoud, "Simulation of Handoff Techniques in Mobile Cellular Networks", Al-Rafidain Engineering, Vol. 15 No.4, 2007, Pp: 31 - 39.

[5] Huamin Zhu and Kyung Sup Kwak, “An Adaptive Hard Handoff Algorithm for Mobile Cellular Communication Systems", ETRI Journal, Volume 28, Number 5, October 2006, Pp: $676-679$.

[6] Sanjay Dhar Roy, "A Timer based Handoff Algorithm for Multi-cellular Systems", ICETET 2008, IEEE, Pp: 819 - 822.

[7] Biswajit Bhowmik, Smita Roy, Parag Kumar Guha Thakurta, Arnab Sarkar, "Priority Based Hard Handoff Management Scheme for Minimizing Congestion Control in Single Traffic Wireless Mobile Networks", International Journal of Advancements in Technology, Vol. 2(1), 2011, Pp: 90-99.

[8] Biswajit Bhowmik, Pooja, Piyali Sarkar, Nupur Thakur, "Received Signal Strength Based Effective Call Scheduling in Wireless Mobile Network", International Journal of Advancements in Technology, Vol. 2(2), 2011, Pp: 292 - 305.

[9] Rajat Prakash, Venugopal V. Veeravalli, "Adaptive Hard Handoff Algorithms”, Proc. VTC'99, Houston, TX, May 99.

[10] Mohmmad Anas, Francesco D. Calabrese, Preben E. Mogensen, Claudio Rosa, Klaus I. Pedersen, "Performance Evaluation of Received Signal Strength Based Hard Handover for UTRAN LTE", IEEE Vehicular Technology Conference, April 2007, Pp:1046 - 1050.

[11] Venugopal V. Veeravalli, Owen E. Kelly, "A Locally optimal handoff algorithm for cellular communications", IEEE Trans. on Vehicular Technology, 1997, Pp: $1-8$.

[12] Sanjay Dhar Roy, "Performance Analysis of Handoff Algorithms for Multihop Ad Hoc Wireless Network", ICETET 2008, IEEE, Pp: 157 - 161.

[13] Biswajit Bhowmik, Pooja, Nupur Thakur, Piyali Sarkar, "Experimental Analysis of Xie and Kuek's Traffic Model with Handoff Scheme in Wireless Networks", Int. J. of Information Engineering and Electronic Business, Vol. 4, No. 1, 2012, Pp: 34-43.

[14] Hua Jiang and Stephen S. Rappaport, "Hand-Off Analysis for CBWL Schemes in Cellular Communications", CEAS Technical Report, No. 683, 1993.

[15] Biswajit Bhowmik, Arnab Sarkar, Parag Kumar Guha Thakurta, "Simulation of Handoff Management Scheme for Improved Priority Based Call Scheduling with a Single Traffic System in Mobile Network", Int. J. of Advanced Research in Computer Science", Vol. 1(3), 2010, Pp: 354-358.

[16]Biswajit Bhowmik, Design and Analysis of Algorithms, S.K. Kataria \& Sons, Second Edition, 2012.

[17] Raymond M. Bendett and Perambur S. Neelakanta, "Alternative Metrics for Hard Handoffs in Mobile Communication", ICPWC 2000, IEEE, 2000, Pp: $43-46$.
[18] J K Sharma, Operations Research - Theory and Application, Macmillan Publishers, 3/e, 2006.

[19] Azita Laily Yusof, Mahamod Ismail, Norbahiah Misran, "Traffic Management Algorithm and Adaptive Handover Initiation Time for Dynamic Traffic Load Distribution", IJCSNS International Journal of Computer Science and Network Security, Vol 8 No7, 2008, Pp: 203 - 207.

[20] Marco Anisetti, Claudio A. Ardagna, Valerio Bellandi Ernesto Damiani, Salvatore Reale, "Advanced Localization of Mobile Terminal in Cellular Network", I. J. Communications, Network and System Sciences, Vol 1, 2008, Pp: 95 - 103.

[21] Nguyen Cao Phuong, Sang-Ho Lee, Jung-Mo Moon, Tran Hong Quan, "Priority-based Call Admission Control of multiclasses in Mobile networks", ICA0T2006, IEEE Xplore, Feb. 20-22, 2006,Pp: $1471-1474$.

[22] Hsin-Piao Lin, Rong-Terng Juang, and Ding-Bing Lin, "Improved Location-Based Handover Algorithm for Mobile Cellular Systems with Verification of GSM Measurements Data", IEEE Trans. on Vehicular Technology, 2002, Pp: $5170-$ 5174.

[23] Thomas H. Cormen, Charles E. Leiserson, Ronald L. Rivest, and Clifford Stein, "Introduction to Algorithms, PHI, 2nd Edition, 2006.

[24] D. Samanta, Classic Data Structures, PHI, 2nd Edition, $18^{\text {th }}$ printing, 2010.

[25]P. K. Guha Thakurta, Souvik Sonar, Biswajit Bhowmik, Swapan Bhattacharya, Subhansu Bandyopadhyay, "A New Approach on Priority Queue based Scheduling with Handoff Management for Mobile Networks", $19^{\text {th }}$ Int. Conf. on SEDE, ISCA, 2010, Pp: $69-74$.

[26] http://www.coai.com/study_papers.php?val=2010

[27] http://en.wikipedia.org/wiki/Kolkata\#Geography.

Biswajit Bhowmik is currently doing $\mathrm{PhD}$ in the Department of Computer Science \& Engineering of Indian Institute of Technology Guwahati. He was a very renowned faculty member with Bengal College of Engineering and Technology, Durgapur, India as Assistant Professor in the Department of Computer Science \& Engineering for almost 7 years. He is a member of different professional bodies such as IEEE, IACSIT, IAENG, IAS, IAOE, ISOC, PASS, UACEE etc. $\mathrm{He}$ is also a member of some professional societies such as IEEE Computer Society, IEEE Communications Societies, IAENG Society of Computer Science, IAENG Society of Wireless Networks, IAENG Society of Software Engineering, and IAENG Society of Artificial Intelligence. $\mathrm{He}$ is reviewer of several international journals such as ETASR, IJCSIC, IJCSIS, JACSM, IJoAT, JWMC etc in the area of computer science, wireless networks, and mobile computing. He has authored a book titled Design and Analysis of Algorithms. $\mathrm{He}$ has many publications in international journals 
including international conference proceedings on the subjects ranging from Algorithms Analysis, Graph Theory, Compiler Design, Wireless Networks, and Mobile Computing. In addition his area of interests includes Data Structures \& Algorithms, Computational Geometry, and Green Computing. He has guided several projects at under graduate level. This paper is based on Wireless Mobile Networks as Final Year Thesis Work for UG Curriculum under West Bengal University of Technology, Kolkata, India.

Pooja, Piyali Sarkar, and Nupur Thakur are Final Year UG (B.Tech) students in the Department of Computer Science \& Engineering at Bengal College of Engineering and Technology, Durgapur, India. They are doing this thesis work as partial fulfillment of the requirements of their B.Tech Degree in Computer Science \& Engineering from the institute affiliated under West Bengal University of Technology, Kolkata. 